\title{
Asymmetric dimethylarginine is not a marker of arterial damage in children with glomerular kidney diseases
}

\author{
PIOTR SKRZYPCZYK ${ }^{1}$, JOANNA PRZYCHODZIEN' ${ }^{1}$, MAEGORZATA MIZERSKA-WASIAK ${ }^{1}$, \\ ELŻBIETA KUŹMA-MROCZKOWSKA', ANNA STELMASZCZYK-EMMEL ${ }^{2}$, ELŻBIETA GÓRSKA2, \\ MAEGORZATA PAŃCZYK-TOMASZEWSKA ${ }^{l}$ \\ ${ }^{1}$ Department of Pediatrics and Nephrology, Medical University of Warsaw, Warsaw, Poland \\ ${ }^{2}$ Department of Laboratory Diagnostics and Clinical Immunology of Developmental Age, Medical University of Warsaw, Warsaw, Poland
}

\begin{abstract}
Introduction: Asymmetric dimethylarginine (ADMA), an endogenous inhibitor of nitric oxide synthase, correlates with cardiovascular risk especially in patients with chronic kidney disease. The aim of our study was to establish significance of ADMA as a biomarker of arterial damage in children with glomerulopathies.

Material and methods: In 80 children with glomerulopathies (mean age, $11.33 \pm 4.25$ years; 42 with idiopathic nephrotic syndrome [INS], 38 with IgA or Henoch-Schoenlein nephropathy [IgAN/HSN]), we analyzed serum ADMA [nmol/ml], peripheral and central blood pressure, arterial stiffness (augmentation index - AIx $75 H R$, pulse wave velocity - PWV), common carotid artery intima media thickness (cIMT), and selected clinical and biochemical parameters.

Results: In the study group, mean ADMA concentration was $1.66 \pm 1.19[\mathrm{nmol} / \mathrm{ml}]$ and did not differ between INS and IgAN/HSN patients. We found no significant correlations between concentration of ADMA, cIMT [mm]/Z-score, PWV [m/s]/Z-score, and AIx75HR [\%] in the whole group and in INS and IgAN/HSN patients. In the whole group of 80 children, ADMA correlated $(p<0.05)$ with BMI Z-score $(r=-0.24)$, uric acid $(r=-0.23)$, HDL-cholesterol $(r=-0.25)$, and central mean arterial pressure $(r=-0.25)$, in children with INS also with total protein $(r=0.37)$, albumin $(r=0.36)$, and total cholesterol $(r=-0.40, p=0.028)$. In multivariate analysis, serum albumin was the strongest determinant of ADMA in the whole group ( $\beta=0.536,95 \%$ CI: 0.013-1.060, $p=0.045)$.

Conclusions: 1 . In children with glomerulonephritis, measurement of asymmetric dimethylarginine cannot replace well established and validated methods of assessment of subclinical arterial damage. 2. In children with glomerular kidney diseases, ADMA concentration is related primarily to serum albumin concentration.
\end{abstract}

Key words: children, asymmetric dimethylarginine, glomerular kidney disease, arterial damage.

(Centr Eur J Immunol 2019; 44 (4): 370-379)

\section{Introduction}

Glomerular kidney diseases are autoimmune disorders manifesting either as asymptomatic proteinuria or erythrocyturia, nephrotic or nephritic syndrome. Adult patients with glomerular kidney diseases were found to have increased cardiovascular morbidity and mortality [1]. In our previous studies we have revealed increased peripheral and central blood pressure [2], increased arterial stiffness $[2,3]$, and increased common carotid artery intima-media thickness $[2,4]$ in pediatric patients with glomerulopathies. The pathophysiology of increased cardiovascular damage in these patients remains uncertain, but may be at least partially caused by increased sympathetic tone, hyperlipidemia, and side effects of the treatment.

Idiopathic nephrotic syndrome (INS) is the most common form of nephrotic syndrome in children characterized by normal renal function and blood pressure, isolated proteinuria, relapsing course, and usually good response to steroids [5]. IgA nephropathy is the most common glomerulonephritis and Henoch-Schönlein purpura is the most common form of vasculitis in pediatric patients. Renal involvement (Henoch-Schönlein nephropathy - HSN) is reported in $20-60 \%$ of affected children. IgAN and HSN

Correspondence: Piotr Skrzypczyk, MD, PhD, Department of Pediatrics and Nephrology, Medical University of Warsaw, 63A Żwirki i Wigury St., 02-091 Warsaw, Poland, e-mail: pskrzypczyk@wum.edu.pl Submitted: 26.07.2019; Accepted: 3.10.2019 
are considered to be different manifestations of one disease with deposition of immune complexes containing immunoglobulin A and with the same galactose-deficient IgA1-oriented pathogenesis [6].

Evaluation of subclinical target-organ damage in children requires expensive equipment, experienced personnel, is time-consuming and, commonly, operator-dependent. Hence, research has been conducted aimed at finding serological markers of increased cardiovascular burden. Adult population studies and pilot pediatric reports suggest that asymmetric dimethylarginine (ADMA) could be one of such biomarkers $[7,8]$.

ADMA is a methylated L-arginine derivative produced by all nucleated cells. The main source of ADMA are L-arginine-rich proteins, previously methylated during post-translational modifications (e.g. histones). Once synthesized, ADMA migrates into the extracellular space and into bloodstream, where it exerts its biological actions. ADMA is eliminated from bloodstream through urine excretion and metabolized by the enzyme dimethylarginine dimethylaminohydrolase (DDAH). ADMA is an endogenous inhibitor of nitric oxide synthesis by competitive inhibition of L-arginine uptake by endothelial cells $[9,10]$. Numerous studies in adult population showed positive relation between ADMA and both intermediate end-points $[7,8]$ as well as cardiovascular morbidity and mortality $[11,12]$. There are limited data on usefulness of ADMA assessment in children with nephrotic syndrome [13], hypertension [14], juvenile idiopathic arthritis [15], and chronic kidney disease [16, 17].

The primary aim of our study was to establish the significance of ADMA as a biomarker of subclinical arterial damage in children with two most common glomerular kidney diseases, INS and IgAN/HSN. The secondary aim was to find a relation between serum ADMA concentration and clinical and biochemical parameters in these patients.

\section{Material and methods}

In this cross-sectional study, we analyzed data of 80 children with glomerular kidney diseases (INS and IgAN/HSN patients) treated at one tertiary center of pediatric nephrology. The enrollment took place between October 2013 and May 2016. The inclusion criteria were: age 5-18 years and biopsy-proven IgAN/HSN or unquestionable clinical diagnosis of INS $[4,5]$. The exclusion criteria were: chronic kidney disease (CKD) stage 4 or 5 according to KDIGO [18], diabetes mellitus, liver, heart, or arterial diseases, and acute infections (temporary exclusion). Patients with INS were examined in the remission of the disease ( $\geq 3$ days without proteinuria), except for drug-resistant patients in whom remission could not have been achieved. The indications to kidney biopsy in INS patients were: age at onset $<1$ year or $>10$ years, steroid resistance, renal dysfunction, and clinical course or biochemical tests suggestive of other glomerulopathy [4, 5].

Venous peripheral blood was collected after overnight fasting, centrifuged to obtain serum, frozen and stored at $-80^{\circ} \mathrm{C}$ until analysis. ADMA concentration was measured in serum using commercially available enzyme-linked immunosorbent assay (ELISA) (DRG Instruments $\mathrm{GmbH}$, Marburg, Germany) (nmol/ml).

Following clinical data were collected: type of glomerular kidney disease: INS/IgAN/HSN, age (years), sex, body mass index (BMI) Z-score [19], disease vintage (year), number of relapses (patients with INS), kidney biopsy findings, presence of arterial hypertension, and medications used. Overweight was defined as BMI Z-score $>1.0$ and obesity as BMI Z-score $>2.0$. Corticosteroid doses were converted to prednisone dose $(\mathrm{mg} / \mathrm{kg} / 24 \mathrm{~h})$. Five grade $\left(\mathrm{I}^{\circ}-\mathrm{V}^{\circ}\right)$ WHO classification was used to evaluate kidney biopsy findings in IgAN/HSN patients [20]. Serum concentrations of creatinine $(\mathrm{mg} / \mathrm{dl})$, uric acid $(\mathrm{mg} / \mathrm{dl})$, total, low-density (LDL) and high-density lipoprotein (HDL) cholesterol (mg/dl), triglycerides $(\mathrm{mg} / \mathrm{dl})$ were assessed. Proteinuria was evaluated in morning urine sample $(\mathrm{mg} / \mathrm{dl})$ and in daily urinary collection $(\mathrm{mg} / \mathrm{kg} / 24 \mathrm{~h})$. Biochemical variables were measured using VITROS 5600 (Ortho Clinical Diagnostics, Linden, NJ, USA). Glomerular filtration rate $\left(\mathrm{ml} / \mathrm{min} / 1.73 \mathrm{~m}^{2}\right)$ was calculated [21] and stages of CKD were classified according to KDIGO classification [18]. Impaired renal function was defined as GFR $<90 \mathrm{ml} / \mathrm{min} / 1.73 \mathrm{~m}^{2}$. Hyperuricemia was defined as uric acid $>6.0 \mathrm{mg} / \mathrm{dl}$, hypoproteinemia as total protein $<6.0 \mathrm{~g} / \mathrm{dl}$, hypoalbuminemia as albumin $<3.5 \mathrm{~g} / \mathrm{dl}$, hypercholesterolemia as cholesterol $\geq 200 \mathrm{mg} / \mathrm{dl}$, and hypertriglyceridemia as triglyceride $\geq 100 \mathrm{mg} / \mathrm{dl}$ (children aged 0-9 years) or $\geq 130 \mathrm{mg} / \mathrm{dl}$ (children aged 10-19 years) [22], and nephrotic proteinuria as daily urinary protein loss $\geq 50 \mathrm{mg} / \mathrm{kg} / 24 \mathrm{~h}$.

Peripheral blood pressure (BP) was measured using Welch Allyn VSM Vital Sings Monitor 300 (Welch Allyn Inc., Skaneateles Falls, NY, USA), and expressed as $\mathrm{mm} \mathrm{Hg}$ and $\mathrm{Z}$-score [23]. Central (aortic) blood pressure (AoBP) ( $\mathrm{mm} \mathrm{Hg}$ ), augmentation index normalized to heart rate of 75 beats per minute (AIx75HR) (\%), subendocardial viability ratio (SEVR) (\%), and aortic pulse wave velocity (PWV) (m/s) were evaluated with Sphygmocor device (AtCor Medical Pty Ltd., Sydney, Australia), and common carotid intima-media thickness (cIMT) (mm) with Aloka Prosound Alpha 6 (Hitachi Aloka Medical, Mitaka, Japan) using 13-MHz linear transducer. Detailed methods of central blood pressure and arterial damage assessment have been described in our previous studies [2, 4]. PWV and cIMT were expressed as absolute values and $Z$-scores [24, 25]. Central systolic blood pressure (AoSBP) was compared with normative data [26]. Peripheral and central BP, PWV, and cIMT $\geq 95^{\text {th }}$ percentile were considered abnormal.

All procedures were in accordance with ethical standards of the Institutional Bioethical Committee (approval 
No. KB/199/2012), and with 1964 Helsinki declaration and its later amendments. Informed consent was obtained from all participants included in the study ( $\geq 16$ years) and their representatives.

Statistical elaboration was performed using Dell Statistica 13.0 PL (Dell Inc., AlisoViejo, CA, USA). Variables were presented as absolute numbers and frequencies or mean \pm standard deviation (SD). Normality of data was analyzed with Shapiro-Wilk test. Following tests were used (depending on data distribution): Student's $t$-test, U Mann-Whitney test, Spearman rank correlation, $\chi^{2}$ test, and Fisher's exact test. Multivariate analysis was performed using general regression model. Parameters that correlated with ADMA concentration in univariate analysis with $p<0.100$ together with markers of arterial damage (PWV Z-score, cIMT Z-score, and AIx75HR) were included in the model. A $p$-value $<0.05$ was considered statistically significant.

\section{Results}

The clinical characteristics of the study group are presented in Table 1. Patients with INS and patients with IgAN/
HSN did not differ in sex, age, and BMI Z-score. Overweight was found in 19 (11 with INS, 8 with IgAN/HSN) and obesity in 17 (12 with INS, 5 with IgAN/HSN) participants. The duration of the disease was longer and prevalence of hypertension was more common in patients with INS. In the INS group, there were 17 steroid-resistant (SR) and 25 steroid-sensitive/steroid-dependent patients (SS/SD). Kidney biopsy was performed in 32/42 patients with INS - minimal change disease was revealed in 4, mesangial proliferation in 22, and focal and segmental glomerulosclerosis in 6 children. In the IgAN/HSN group there were 16 patients with IgAN and 22 with HSN. Classification of kidney biopsy results according to WHO classification in IgAN/HSN group was as follows: 1 patient with class 1,14 patients with class 2, 19 class 3, and 4 with class 4 . Corticosteroids were used in comparable percentage of patients from both groups, but corticosteroid dose was significantly higher in IgAN/HSN group. There were also significant differences in usage of other immunosuppressive medications resulting from disease-specific protocols. Biochemical parameters are depicted in Table 2. The groups did not differ in GFR. Impaired renal function was found in 15 patients: CKD stage 2 was revealed in 13 children (7 with INS and 6 with IgAN/

Table 1. Clinical characteristics of the studied children

\begin{tabular}{|c|c|c|c|c|}
\hline Characteristics & Study group & INS & IgAN/HSN & $p^{1}$ \\
\hline Number of patients & 80 & 42 & 38 & \\
\hline Boys/girls & $50 / 30$ & $23 / 19$ & $27 / 11$ & 0.133 \\
\hline Age, years & $11.33 \pm 4.25$ & $10.81 \pm 4.36$ & $11.91 \pm 4.09$ & 0.219 \\
\hline Duration of the disease, years & $4.71 \pm 4.36$ & $6.78 \pm 4.35$ & $2.46 \pm 3.06$ & $<0.001$ \\
\hline Number of relapses (INS) & - & $8.11 \pm 5.88$ & - & - \\
\hline BMI Z-score & $0.87 \pm 1.09$ & $0.96 \pm 1.17$ & $0.77 \pm 1.011$ & 0.850 \\
\hline Arterial hypertension, $n(\%)$ & $35(43.8)$ & $23(54.8)$ & $12(31.6)$ & 0.037 \\
\hline \multicolumn{5}{|l|}{ Antihypertensive medications, $n$} \\
\hline $\mathrm{ACEi}$ & 60 & 31 & 29 & 0.796 \\
\hline $\mathrm{CCB}$ & 11 & 5 & 6 & 0.614 \\
\hline $\mathrm{BB}$ & 5 & 5 & 0 & 0.056 \\
\hline HCTZ & 1 & 1 & 0 & 1.000 \\
\hline Spironolactone & 1 & 1 & 0 & 1.000 \\
\hline $\mathrm{GC}, n$ & 60 & 33 & 27 & 0.453 \\
\hline $\mathrm{GC}, \mathrm{mg} / \mathrm{kg} / 24 \mathrm{~h}$ & $0.58 \pm 0.58$ & $0.39 \pm 0.45$ & $0.79 \pm 0.65$ & 0.006 \\
\hline \multicolumn{5}{|l|}{ Immunosuppressant agents } \\
\hline Cyclosporine & 25 & 23 & 2 & $<0.001$ \\
\hline Azathioprine & 11 & 0 & 11 & $<0.001$ \\
\hline Mycophenolate mofetil & 12 & 12 & 0 & $<0.001$ \\
\hline Cyclophosphamide & 4 & 2 & 2 & 1.000 \\
\hline Tacrolimus & 1 & 1 & 0 & 1.000 \\
\hline
\end{tabular}


Table 2. Biochemical data of the studied children

\begin{tabular}{lcccc}
\hline Parameter & Study group & INS & IgAN/HSN & $\boldsymbol{p}$ (INS vs. IgAN/HSN) \\
\hline $\mathrm{GFR}_{\mathrm{s}}, \mathrm{ml} / \mathrm{min} / 1.73 \mathrm{~m}^{2}$ & $109.29 \pm 24.50$ & $109.12 \pm 17.64$ & $109.47 \pm 30.63$ & 0.627 \\
\hline Uric acid, $\mathrm{mg} / \mathrm{dl}$ & $5.31 \pm 1.59$ & $5.65 \pm 1.63$ & $4.92 \pm 1.46$ & 0.044 \\
\hline Total protein, g/dl & $6.79 \pm 0.90$ & $6.67 \pm 0.85$ & $6.89 \pm 0.94$ & 0.215 \\
\hline Albumin, $\mathrm{g} / \mathrm{dl}$ & $4.01 \pm 0.71$ & $4.03 \pm 0.75$ & $3.99 \pm 0.67$ & 0.705 \\
\hline Total cholesterol, $\mathrm{mg} / \mathrm{dl}$ & $224.97 \pm 88.52$ & $243.55 \pm 97.30$ & $209.82 \pm 78.73$ & 0.098 \\
\hline HDL cholesterol, $\mathrm{mg} / \mathrm{dl}$ & $68.91 \pm 22.82$ & $72.62 \pm 26.22$ & $65.91 \pm 19.49$ & 0.468 \\
\hline LDL cholesterol, $\mathrm{mg} / \mathrm{dl}$ & $127.55 \pm 74.42$ & $140.21 \pm 76.70$ & $117.62 \pm 72.06$ & 0.113 \\
\hline Triglycerides, $\mathrm{mg} / \mathrm{dl}$ & $140.92 \pm 91.14$ & $151.40 \pm 99.37$ & $132.65 \pm 84.51$ & 0.345 \\
\hline Proteinuria, $\mathrm{mg} / \mathrm{dl}$ & $83.24 \pm 174.70$ & $60.93 \pm 148.90$ & $107.97 \pm 198.62$ & 0.477 \\
\hline Proteinuria, $\mathrm{mg} / \mathrm{kg} / 24 \mathrm{~h}$ & $22.00 \pm 39.33$ & $13.75 \pm 34.85$ & $31.58 \pm 42.53$ & 0.004
\end{tabular}

INS - idiopathic nephrotic syndrome, IgAN - IgA nephropathy, HSN - Henoch-Schönlein nephropathy, GFR - glomerular filtration rate according to Schwartz formula, $H D L$ - high-density lipoprotein, $L D L$-low-density lipoprotein

Table 3. Asymmetric dimethylarginine, peripheral, and central blood pressure and markers of arterial damage in children with glomerular kidney diseases

\begin{tabular}{lcccc}
\hline Parameter & Study group & INS & IgAN/HSN & $\boldsymbol{p}$ (INS vs. IgAN/HSN) \\
\hline ADMA, nmol/ml & $1.66 \pm 1.21$ & $1.72 \pm 1.24$ & $1.60 \pm 1.19$ & 0.958 \\
\hline SBP, $\mathrm{mm} \mathrm{Hg}$ & $114.51 \pm 12.27$ & $114.20 \pm 11.53$ & $114.89 \pm 13.34$ & 0.826 \\
SBP Z-score & $0.93 \pm 1.18$ & $1.11 \pm 1.09$ & $0.70 \pm 1.27$ & 0.181 \\
\hline DBP, $\mathrm{mm} \mathrm{Hg}$ & $70.39 \pm 10.72$ & $70.37 \pm 10.91$ & $70.41 \pm 10.69$ & 0.868 \\
DBP Z-score & $0.84 \pm 0.92$ & $0.94 \pm 0.96$ & $0.72 \pm 0.87$ & 0.256 \\
\hline AoSBP, mm Hg & $98.21 \pm 11.40$ & $98.23 \pm 11.93$ & $98.19 \pm 10.91$ & 0.988 \\
\hline AoDBP, $\mathrm{mm} \mathrm{Hg}$ & $72.06 \pm 10.77$ & $72.29 \pm 10.86$ & $71.77 \pm 10.85$ & 0.853 \\
\hline AoMAP, mm Hg & $85.32 \pm 10.71$ & $85.78 \pm 10.89$ & $84.74 \pm 10.65$ & 0.704 \\
\hline AoPP, $\mathrm{mm} \mathrm{Hg}$ & $26.89 \pm 6.93$ & $27.27 \pm 8.22$ & $26.42 \pm 4.96$ & 0.945 \\
\hline AIx75HR, $\%$ & $8.09 \pm 18.59$ & $11.34 \pm 18.59$ & $4.05 \pm 18.15$ & 0.146 \\
\hline SEVR, $\%$ & $143.28 \pm 40.72$ & $137.50 \pm 34.26$ & $150.50 \pm 47.24$ & 0.284 \\
\hline PWV, m/s & $4.90 \pm 0.75$ & $4.80 \pm 0.68$ & $5.02 \pm 0.82$ & 0.251 \\
\hline PWV Z-score & $0.44 \pm 1.57$ & $0.48 \pm 1.51$ & $0.39 \pm 1.68$ & 0.822 \\
\hline cIMT, mm & $0.45 \pm 0.06$ & $0.45 \pm 0.05$ & $0.45 \pm 0.07$ & 0.948 \\
\hline cIMT Z-score & $1.44 \pm 1.17$ & $1.53 \pm 1.14$ & $1.30 \pm 1.23$ & 0.461 \\
\hline
\end{tabular}

$\overline{I N S}$ - idiopathic nephrotic syndrome, IgAN - IgA nephropathy, HSN - Henoch-Schönlein nephropathy, ADMA - asymmetric dimethylarginine, SBP - systolic blood pressure, DBP - diastolic blood pressure, AoSBP - aortic (central) systolic blood pressure, AoDBP - aortic (central) diastolic blood pressure, AoMAP - aortic (central) mean blood pressure, AoPP - aortic (central) pulse pressure, AIx75HR - augmentation index normalized to heart rate of 75 beats per minute, SEVR subendocardial viability ratio, $P W V$ - pulse wave velocity, cIMT - common carotid artery intima-media thickness

HSN) and CKD stage 3 in 2 children, with IgAN/HSN and GFR 41.60 and $57.48 \mathrm{ml} / \mathrm{min} / 1.73 \mathrm{~m}^{2}$, respectively. Uric acid was significantly higher in INS children; hyperuricemia was found in 16 patients with INS and 11 with IgAN/HSN. The groups did not differ in terms of total protein, albumin, and lipids. Hypoproteinemia was found in 9 patients (5 with INS, 4 with IgAN/HSN), hypoalbuminemia in 13 (7 with INS, 6 with $\operatorname{IgAN} / \mathrm{HSN}$ ), hypercholesterolemia in 34 (17 with INS, 17 with IgAN/HSN), and hypertriglyceridemia in 30 ( 15 with INS, 15 with IgAN/HSN). At the moment of evaluation, 22 patients were proteinuric ( 7 with INS, 15 with
IgAN/HSN) and 11 had nephrotic proteinuria (2 with INS, 9 with $\operatorname{IgAN} / \mathrm{HSN})$. Mean daily proteinuria was higher in IgAN/HSN group.

Concentration of ADMA, peripheral and central blood pressure as well as markers of arterial damage are presented in Table 3. Mean ADMA concentration was comparable in both groups of the patients (Fig. 1). Patients with INS did not differ compared to IgAN/HSN patients in peripheral and central blood pressure, arterial stiffness, and cIMT. At the moment of evaluation, elevated peripheral systolic blood pressure was revealed in 11 subjects (6 with INS, 


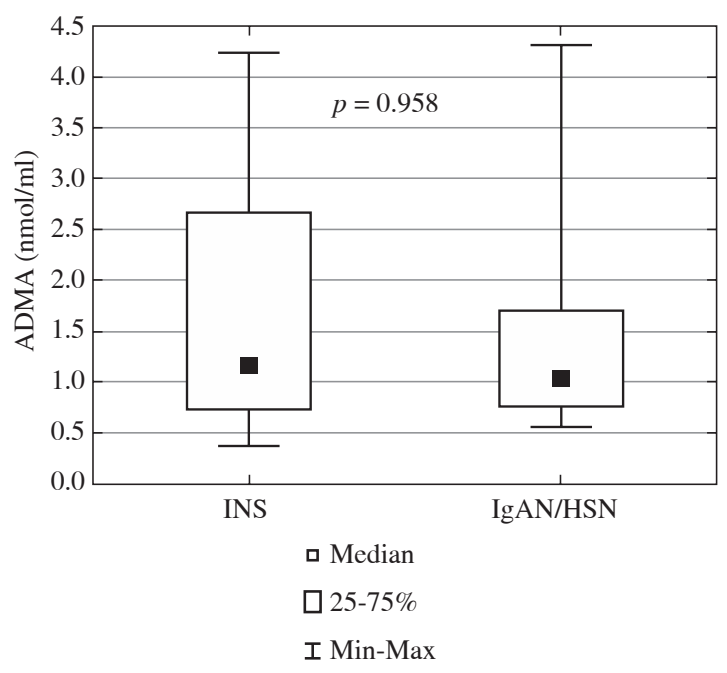

Fig. 1. Asymmetric dimethylarginine in children with idiopathic nephrotic syndrome and IgA nephropathy/Henoch-Schönlein nephropathy (ADMA - asymmetric dimethylarginine, INS - idiopathic nephrotic syndrome, IgAN/ HSN - IgA nephropathy/Henoch-Schönlein nephropathy)

with $5 \operatorname{Ig} \mathrm{AN} / \mathrm{HSN})$, peripheral diastolic blood pressure in 6 (4 with INS, 2 with IgAN/HSN), and elevated central systolic blood pressure in 10 (5 with INS, 5 with IgAN/ HSN). Abnormal PWV was revealed in 9 (6 with INS, 3 with IgAN/HSN) and abnormal cIMT in 20 patients (11 with INS, 9 with IgAN/HSN).

In the whole group of 80 patients, ADMA concentration did not differ between boys and girls $(1.72 \pm 1.24$ vs. $1.56 \pm 1.17[\mathrm{nmol} / \mathrm{ml}], p=0.777)$, between patients with and without arterial hypertension $(1.62 \pm 1.18$ vs. 1.69 $\pm 1.24[\mathrm{nmol} / \mathrm{ml}], p=0.742$ ), and between those with and without proteinuria $(1.59 \pm 1.21$ vs. $1.69 \pm 1.22[\mathrm{nmol} / \mathrm{ml}$ ], $p=0.616)$. Patients treated with corticosteroids had significantly lower ADMA concentration compared to those who were not receiving corticosteroids $(1.51 \pm 1.16$ vs. $2.12 \pm 1.27$ [nmol/ml], $p=0.017$ ) (Fig. 2). There was no difference between those treated and not treated with ACEi $(1.60 \pm 1.16$ vs. $1.84 \pm 1.37[\mathrm{nmol} / \mathrm{ml}], p=0.894)$. Also, no relation between ADMA concentration and response to steroids (SS/SD vs. SR), result of kidney biopsy, sex, presence of arterial hypertension, presence of proteinuria, treatment with cyclosporine A, mofetil mycophenolate, and ACEi was found in children with INS $(p=0.818$, $p=0.285, p=0.324, p=0.390, p=0.380, p=0.331$, $p=0.358$, and $p=0.290$, respectively). Children with INS treated with corticosteroids had significantly lower ADMA compared to those who were not treated $(1.55 \pm 1.19$ vs. $2.38 \pm 1.29[\mathrm{nmol} / \mathrm{ml}], p=0.040)$. In IgAN/HSN, no relation between ADMA and extent of disease (renal limited - IgAN vs. systemic - HSN), WHO classification, sex, presence of arterial hyper-

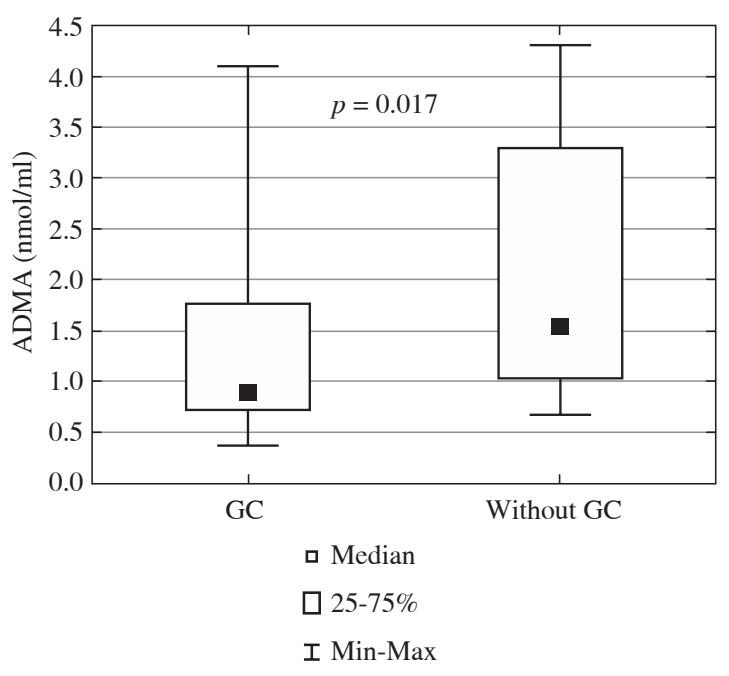

Fig. 2. Asymmetric dimethylarginine in children with glomerular kidney diseases treated and not treated with glucocorticoids (ADMA - asymmetric dimethylarginine, $\mathrm{GC}$ - glucocorticoid)

tension, presence of proteinuria, treatment with corticosteroids, azathioprine, and ACEi was revealed $(p=0.712, p=0.562, p=0.342, p=0.572, p=0.823$, $p=0.240, p=0.723$, and $p=0.250$, respectively).

Correlations of ADMA with clinical and biochemical parameters and with blood pressure and markers of arterial damage are presented in Table 4 . In the whole group, we found negative correlations of ADMA with BMI Z-score, uric acid, HDL-cholesterol, and central mean arterial pressure. In addition, in patients with INS, ADMA correlated positively with total protein and albumin, and negatively with total cholesterol. ADMA showed no correlation with renal function (GFR). Also, no correlation was found when we analyzed only those 15 individuals with impaired renal function $(r=0.09, p=0.796)$. No association was revealed between ADMA and corticosteroid dose in the treated patients. In whole group and also in subgroups of patients with INS and IgAN/HSN, no significant correlations were found between serum ADMA concentration and markers of arterial damage: AIx, PWV, and cIMT. Similarly, no significant correlations between ADMA and aforementioned markers were revealed in subgroups of proteinuric and non-proteinuric patients. Results of multivariate analysis are reported in Table 5. Serum albumin was the strongest determinant of ADMA in patients with glomerular kidney diseases $(\beta=0.536,95 \%$ CI: $0.013-1.060, p=0.045)$.

\section{Discussion}

Results of our study did not show any significant relation between ADMA concentration and either thickening 
Table 4. Correlations of ADMA in children with glomerular kidney diseases with selected clinical and biochemical parameters, with blood pressure and markers of arterial damage

\begin{tabular}{|c|c|c|c|}
\hline \multirow[t]{2}{*}{ Analyzed parameter } & \multicolumn{3}{|c|}{ Correlation with ADMA $(R, p)$} \\
\hline & Study group & INS & IgAN/HSN \\
\hline Age, years & $-0.07,0.522$ & $-0.11,0.481$ & $-0.11,0.530$ \\
\hline Duration of the disease, $n$ & $0.06,0.613$ & $0.00,0.999$ & $0.08,0.619$ \\
\hline Number of relapses (INS) & - & $-0.04,0.804$ & - \\
\hline BMI Z-score & $-0.24,0.035$ & $-0.23,0.150$ & $-0.24,0.155$ \\
\hline $\mathrm{CS}, \mathrm{mg} / \mathrm{kg} / 24 \mathrm{~h}$ & $-0.10,0.463$ & $-0.16,0.379$ & $0.03,0.880$ \\
\hline $\mathrm{GFR}_{\mathrm{S}}, \mathrm{ml} / \mathrm{min} / 1.73 \mathrm{~m}^{2}$ & $0.15,0.184$ & $0.20,0.212$ & $0.21,0.202$ \\
\hline Uric acid, mg/dl & $-0.23,0.042$ & $-0.35,0.024$ & $-0.15,0.377$ \\
\hline Total protein, g/dl & $0.19,0.102$ & $0.36,0.030$ & $-0.04,0.820$ \\
\hline Albumin, $\mathrm{g} / \mathrm{dl}$ & $0.21,0.071$ & $0.37,0.022$ & $-0.03,0.877$ \\
\hline Total cholesterol, mg/dl & $-0.21,0.078$ & $-0.40,0.028$ & $-0.05,0.781$ \\
\hline HDL cholesterol, mg/dl & $-0.25,0.045$ & $-0.32,0.085$ & $-0.16,0.332$ \\
\hline LDL cholesterol, mg/dl & $-0.09,0.473$ & $-0.32,0.090$ & $0.13,0.432$ \\
\hline Triglycerides, mg/dl & $-0.23,0.061$ & $-0.32,0.083$ & $-0.09,0.595$ \\
\hline Proteinuria, mg/dl & $-0.01,0.898$ & $-0.09,0.564$ & $0.05,0.763$ \\
\hline Proteinuria, $\mathrm{mg} / \mathrm{kg} / 24 \mathrm{~h}$ & $0.04,0.763$ & $-0.03,0.846$ & $0.06,0.729$ \\
\hline SBP, mm Hg & $-0.18,0.156$ & $-0.21,0.218$ & $-0.16,0.427$ \\
\hline SBP Z-score & $-0.20,0.118$ & $-0.22,0.199$ & $-0.16,0.438$ \\
\hline DBP, mm Hg & $-0.14,0.286$ & $-0.17,0.323$ & $-0.11,0.569$ \\
\hline DBP Z-score & $-0.09,0.464$ & $-0.08,0.651$ & $-0.10,0.605$ \\
\hline AoSBP, mm Hg & $-0.22,0.081$ & $-0.20,0.245$ & $-0.28,0.146$ \\
\hline AoDBP, mm Hg & $-0.20,0.109$ & $-0.20,0.260$ & $-0.20,0.317$ \\
\hline AoMAP, mm Hg & $-0.25,0.045$ & $-0.25,0.154$ & $-0.24,0.218$ \\
\hline AoPP, mm Hg & $-0.12,0.339$ & $-0.20,0.238$ & $0.00,0.982$ \\
\hline AIx75HR, \% & $-0.16,0.226$ & $-0.22,0.238$ & $0.01,0.965$ \\
\hline SEVR, \% & $0.13,0.295$ & 0.11 .0 .547 & $0.16,0.424$ \\
\hline $\mathrm{PWV}, \mathrm{m} / \mathrm{s}$ & $-0.04,0.747$ & $-0.03,0.870$ & $-0.10,0.608$ \\
\hline PWV Z-score & $-0.08,0.557$ & $0.03,0.874$ & $-0.20,0.321$ \\
\hline cIMT, mm & $0.03,0.795$ & $0.10,0.580$ & $-0.12,0.562$ \\
\hline cIMT Z-score & $0.01,0.946$ & $0.09,0.930$ & $-0.13,0.538$ \\
\hline
\end{tabular}

INS - idiopathic nephrotic syndrome, IgAN - IgA nephropathy, HSN - Henoch-Schönlein nephropathy, ADMA - asymmetric dimethylarginine, BMI - body mass index, GC-glucocorticoid, GFR - glomerular filtration rate according to Schwartz formula, $H D L$ - high-density lipoprotein, LDL - low-density lipoprotein, $S B P$ - systolic blood pressure, DBP - diastolic blood pressure, AoSBP - aortic (central) systolic blood pressure, AoDBP - aortic (central) diastolic blood pressure, AoMAP - aortic (central) mean blood pressure, AoPP - aortic (central) pulse pressure, AIx $75 H R$ - augmentation index normalized to heart rate of 75 beats per minute, SEVR - subendocardial viability ratio, $P W V$ - pulse wave velocity, cIMT - common carotid artery intima-media thickness

of arterial wall (cIMT) or parameters of arterial stiffness (PWV and AIx75HR) in both univariate and multivariate analysis. We found negative correlation of ADMA with central mean arterial pressure, BMI Z-score, and uric acid. In addition, in patients with INS, ADMA correlated with typical biochemical disturbances: positively with total protein and albumin, and negatively with total cholesterol. Serum albumin concentration was the strongest predictor of ADMA.
We did not find significant relations between ADMA and sex or age. ADMA concentration was inversely related to BMI Z-score in univariate model. In pediatric patients with primary hypertension, ADMA was positively related to waist-hip ratio and was significantly higher in those with metabolic syndrome, though no direct correlation with BMI was shown [14]. In another study, ADMA was positively associated with BMI Z-score in hyperuricemic adolescents [27]. On the contrary, Japanese authors observed 
Table 5. Multivariate analysis of factors influencing concentration of ADMA including markers of arterial damage

\begin{tabular}{lccc}
\hline Analyzed parameter & $\beta$ & $\mathbf{9 5 \%}$ confidence interval & $\boldsymbol{p}$ \\
\hline Albumin, g/dl & 0.536 & $0.013-1.060$ & 0.045 \\
\hline BMI Z-score & -0.386 & $-0.808-0.035$ & 0.071 \\
\hline Alx75HR, \% & -0.277 & $-0.682-0.127$ & 0.170 \\
\hline cIMT Z-score & -0.194 & $-0.570-0.183$ & 0.299 \\
\hline AoMAP, mm Hg & -0.493 & $-1.528-0.543$ & 0.336 \\
\hline PWV Z-score & 0.194 & $-0.227-0.616$ & 0.351 \\
\hline Total cholesterol, mg/dl & 0.251 & $-0.597-1.099$ & 0.548 \\
\hline AoSBP, mm Hg & 0.186 & $-0.723-1.096$ & 0.676 \\
\hline Uric acid, mg/dl & 0.090 & $-0.354-0.534$ & 0.679 \\
\hline Triglycerides, mg/dl & 0.120 & $-0.477-0.718$ & 0.682 \\
\hline Glucocorticoids (yes/no) & -0.07 & $-0.544-0.407$ & 0.769 \\
\hline HDL cholesterol, mg/dl & -0.01 & $-0.416-0.393$ & 0.953 \\
\hline ADMA
\end{tabular}

ADMA - asymmetric dimethylarginine, BMI - body mass index, AIx $75 H R$ - augmentation index normalized to heart rate of 75 beats per minute, cIMT - common carotid artery intima-media thickness, AoMAP - aortic (central) mean blood pressure, PWV-pulse wave velocity, AoSBP-aortic (central) systolic blood pressure, $H D L$ - high-density lipoprotein

significantly lower ADMA in obese patients compared to lean children [28]. There is a strong pathophysiological link between elevated serum uric acid and endothelial dysfunction. Uric acid was shown to damage directly endothelial cells. Both uric acid and ADMA were found to decrease NO production in these cells [29, 30]. Indeed, a positive correlation between serum ADMA and uric acid was depicted in pediatric hyperuricemic patients [27]. On contrary, uric acid was inversely related to ADMA in our cohort. It is possible that the relation between body mass, uric acid, and ADMA is overridden in patients with glomerulopathies by other factors (e.g. dysproteinemia), especially in case of immunosuppressive treatment.

Kidneys provide a significant route for clearance of methylarginines. Thus, it is not surprising that ADMA concentrations increase in patients with CKD $[8,9]$. In our cohort, we have not found any significant association between serum ADMA level and GFR. This discrepancy may be due to the fact that only small subgroup of our subjects showed mild to moderate impairment of renal function and we excluded patients with CKD stages 4 and 5. Similarly to our results, no difference was found in terms of ADMA level between pediatric patients with normal kidney function and CKD stages 2 and 3 [16].

In 42 patients with nephrotic syndrome, ADMA was positively related to level of proteins and negatively to total cholesterol. Additionally, in multivariate analysis, albumin level was the strongest predictor of ADMA in the whole group. It must be emphasized that most of our patients with INS were in remission of the disease. Nevertheless, many of them were tested within a month after last relapse and had slightly lowered albumin and elevated cholesterol. Our results are in accordance with the study by Hyla-Klekot who found positive correlations of ADMA, with total protein (in remission phase) and negative correlations with triglycerides and cholesterol (in relapse phase) [13]. In physiological state, $90 \%$ of ADMA is bound to albumin [10]. Thus, loss of albumins would directly impact total ADMA blood concentration, similarly to other albumin-bound small-molecular-weight molecules.

In contrast to numerous population studies in adults [11], we found negative correlation between ADMA and cholesterol. It is hypothesized that high-concentrations of LDL, especially ox-LDL, increase expression of the methyltransferase gene (responsible for ADMA synthesis) and suppression of DDAH activity [10]. It is probable that in patients with glomerulopathies, protein disturbances override this mechanism. Hyla-Klekot suggested also that liver hyperactivity, observed in INS, promotes expression of DDAH [13]. This would strengthen negative correlation between cholesterol (and apolipoproteins) and ADMA.

We did not find any relation between extent of protein loss and ADMA in the studied children as well as in subgroups with INS and IgAN/HSN. This finding supports thesis that ADMA is dependent rather on serum protein concentration, not on its loss. On the contrary, Yilmaz et al. found strong positive correlation $(r=0.88, p<0.001)$ between ADMA and daily urinary protein loss [31]. It is noteworthy that those patients were also characterized by hypoalbuminemia, and relation between ADMA and serum albumin was not analyzed.

We found significantly lower ADMA levels in patients treated with GC, though this relation was not dose-dependent and was not confirmed in multivariate model. Similar results were found in children with Duchenne muscular dystrophy [32]. On the contrary, Japanese authors showed 
significant rise in ADMA concentration in 14 young adult individuals with IgAN, treated with GC pulse therapy [33]. Glucocorticoids exert cardiovascular and neuronal protection through a non-transcriptional activation of endothelial nitric oxide synthase (eNOS) in mice [34]. In contrast, GC excess may induce superoxide production in vascular endothelial cells and elicits vascular endothelial dysfunction. It is hypothesized that GCs may affect endothelial function either favorably or harmfully, depending on dose and route of administration. Angiotensin converting enzyme inhibitors are antihypertensive drugs with potent vasoprotective actions, and were found to decrease ADMA in adults with CKD [35]. In our cohort, there was no difference in ADMA level between those treated and not treated with these medications. This negative finding could be explained by small number of untreated patients $(n=20)$, short treatment duration, and lack of prospective analysis of the subjects.

In general population, ADMA levels are independently associated with endothelial dysfunction [36], subclinical arterial damage [7, 37, 38], and all-cause mortality [12]. ADMA was found to correlate positively with arterial stiffness in adult patients with hypertension (AIx75HR) [37], prediabetes (PWV) [38], and in elderly general population (AIx75HR) [7]. As ADMA levels increase in $\mathrm{CKD}$ as a consequence of impaired renal clearance, this negative relation should strengthen alongside with kidney function decline. Indeed, meta-analysis of studies in adult patients with CKD showed strong positive correlation between ADMA and cIMT [8]. Finally, Zoccali showed that ADMA is the second strongest (after age) predictor of allcause and cardiovascular mortality in adults with end-stage renal disease [11].

In our study group, we found no associations between ADMA and any of the measured markers of subclinical arterial damage. It must be stressed that our patients had already been affected by cardiovascular disease, as $43.8 \%$ of them had arterial hypertension, 20\% had abnormally thickened cIMT, and $11.3 \%$ abnormal PWV. Results of our previous studies suggest that in this group of patients, other factors like number of INS relapses, disease vintage, cumulative steroid dose, body mass index, presence of arterial hypertension, and maybe overexpression of renalase, influence arterial damage [2-4]. Our results do not support usage of ADMA as a marker of arterial damage in pediatric patients with glomerular kidney diseases. It is worth to mention that significance of ADMA as a biomarker of vascular damage in pediatric population remains questionable. Although ADMA was significantly and independently associated with cIMT in a group of 405 8-year-old Australian children [39], this relation was not confirmed in high-risk patients. ADMA level did not correlate with cIMT in pediatric patients with primary hypertension [14], with cIMT, PWV, and ambulatory arterial stiffness index (AASI) in children with CKD stages 1-3
[16], with cIMT in children with CKD stages 2-5 [17], or with cIMT, PWV, and AIx75HR in children with juvenile idiopathic arthritis [15].

Interestingly, in our cohort, ADMA correlated negatively with mean central blood pressure. Central blood pressure is considered a better predictor of cardiovascular events in comparison to peripheral blood pressure, as vital organs (heart, kidneys, and brain) are exposed to aortic pressure. An experimental study demonstrated that intravenously dosed ADMA could significantly increase blood pressure [40]. On the other hand, parallel to our results, ADMA concentration was inversely related to blood pressure, vascular resistance, and PWV in young healthy population from young Finns study. The authors suggest that in the young people, ADMA could be a beneficial molecule responsible for maintaining appropriate vascular resistance [41]. Finally, negative correlations of ADMA with uric acid, BMI Z-score, and central mean arterial pressure might suggest even a protective role of this molecule in children with glomerular kidney diseases. Similarly, lower ADMA was found in children with diabetes mellitus type 1 compared to healthy peers $[28,42]$. In these patients, Huemer observed negative correlation of ADMA with blood pressure, similar as in our cohort [42]. The relationship between ADMA and cardiovascular physiology in the young is not yet fully understood and requires further studies.

Several limitations of our study should be discussed. Firstly, we have not analyzed ADMA concentrations in healthy individuals. Secondly, the cross-sectional nature of the study prevents firm conclusions on causal relationship between ADMA and analyzed parameters. Also, the study group was not large and not uniform in terms of treatment and disease vintage.

\section{Conclusions}

In children with glomerulonephritis, the measurement of asymmetric dimethylarginine cannot replace well established and validated methods of assessment of subclinical arterial damage.

In children with glomerular kidney diseases, ADMA concentration is related primarily to serum albumin concentration.

\section{The authors declare no conflict of interest.}

\section{References}

1. Gustafsson JT, Herlitz Lindberg M, Gunnarsson I, et al. (2017): Excess atherosclerosis in systemic lupus erythematosus - a matter of renal involvement: Case control study of 281 SLE patients and 281 individually matched population controls. PLoS One 12: e0174572.

2. Skrzypczyk P, Przychodzień J, Mizerska-Wasiak M, et al. (2017): Renalase in Children with Glomerular Kidney Diseases. Adv Exp Med Biol 1021: 81-92. 
3. Skrzypczyk P, Mizerska-Wasiak M, Jerszow B, et al. (2017): Ambulatory arterial stiffness index, blood pressure variability, and nocturnal blood pressure dip in children with $\operatorname{IgA}$ and Henoch-Schönlein nephropathy. Clin Nephrol 81: 301-309.

4. Skrzypczyk P, Kuźma-Mroczkowska E, Kułagowska J, et al. (2019): Carotid intima-media thickness in children with idiopathic nephrotic syndrome: a single center cross-sectional study. Clin Nephrol 91: 353-362.

5. Kidney Disease: Improving Global Outcomes (KDIGO) Glomerulonephritis Work Group (2012): KDIGO Clinical Practice Guideline for Glomerulonephritis. Kidney Int Suppl 2: 139-274.

6. Suzuki H, Yasukate J, Makita Y, et al. (2019): IgA nephropathy and IgA vasculitis with nephritis have a shared feature involving galactose-deficient IgA1-oriented pathogenesis. Kidney Int 93: 700-705.

7. Ngo DT, Sverdlov AL, McNeil JJ, Horowitz JD (2009): Correlates of arterial stiffness in an ageing population: Role of asymmetric dimethylarginine. Pharmacol Res 60: 503-507.

8. Wang F, Xiong R, Feng S, et al. (2018): Association of Circulating Levels of ADMA with Carotid Intima-Media Thickness in Patients with CKD: a Systematic Review and Meta-Analysis. Kidney Blood Press Res 43: 25-33.

9. Liu X, Xu X, Shang R, Chen Y (2018): Asymmetric dimethylarginine (ADMA) as an important risk factor for the increased cardiovascular diseases and heart failure in chronic kidney disease. Nitric Oxide 78: 113-120.

10. Morris SM Jr (2016): Arginine metabolism revisited. J Nutr 146 (Suppl): 2579-2586.

11. Zoccali C, Bode-Boger S, Mallamaci F, et al. (2001): Plasma concentration of asymmetrical dimethylarginine and mortality in patients with end-stage renal disease: a prospective study. Lancet 358: 2113-2117.

12. Zhou S, Zhu Q, Li X, et al. (2017): Asymmetric dimethylarginine and all-cause mortality: a systematic review and meta-analysis. Sci Rep 7: 44692.

13. Hyla-Klekot L, Bryniarski P, Pulcer B, et al. (2015): Dimethylarginines as risk markers of atherosclerosis and chronic kidney disease in children with nephrotic syndrome Adv Clin Exp Med 24: 307-313.

14. Śladowska-Kozłowska J, Litwin M, Niemirska A, et al. (2012): Oxidative stress in hypertensive children before and after 1 year of antihypertensive therapy. Pediatr Nephrol 27: 1943-1951.

15. Ilisson J, Zagura M, Zilmer K, et al. (2015): Increased carotid artery intima-media thickness and myeloperoxidase level in children with newly diagnosed juvenile idiopathic arthritis. Arthritis Res Ther 17:180.

16. Chien SJ, Lin IC, Hsu CN, et al. (2015): Homocysteine and arginine-to-asymmetric dimethylarginine ratio associated with blood pressure abnormalities in children with early chronic kidney disease. Circ J 79: 2031-2037.

17. Makulska I, Szczepańska M, Dorota Drożdż D, et al. (2015): Skin autofluorescence as a novel marker of vascular damage in children and adolescents with chronic kidney disease. Pediatr Nephrol 30: 811-819.

18. Kidney Disease: Improving Global Outcomes (KDIGO) CKD Work Group (2013): KDIGO 2012 Clinical Practice Guideline for the Evaluation and Management of Chronic Kidney Disease. Kidney Int Suppl 3: 259-305.

19. Kułaga Z, Litwin M, Tkaczyk M, et al. (2011): Polish 2010 growth references for school-aged children and adolescents. Eur J Pediatr 170: 599-609.
20. Schena FP, Coppo R (2004): IgA nephropathies. In: Oxford Textbook of Clinical Nephrology. Davison AM, Cameron JS, Grunfeld JP, et al. (eds). Oxford University, New York: 470-501.

21. Schwartz GJ, Muńoz A, Schneider MF, et al. (2009): New equations to estimate GFR in children with CKD. J Am Soc Nephrol 20: 629-637.

22. Expert panel on integrated guidelines for cardiovascular health and risk reduction in children and adolescents; National Heart, Lung and Blood Institute (2011): Expert panel on integrated guidelines for cardiovascular health and risk reduction in children and adolescents: summary report. Pediatrics 128 (Suppl 5): 213-256.

23. Kułaga Z, Litwin M, Grajda A, et al. (2012): Oscillometric blood pressure percentiles for Polish normal-weight schoolaged children and adolescents. J Hypertens 30: 1942-1954.

24. Thurn D, Doyon A, Sözeri B, et al. (2015): Aortic pulse wave velocity in healthy children and adolescents: reference values for the Vicorder device and modifying factors. Am J Hypertens 28: 1480-1488.

25. Doyon A, Kracht D, Bayazit AK, et al. (2013): Carotid artery intima-media thickness and distensibility in children and adolescents: reference values and role of body dimensions. Hypertension 62: 550-556.

26. Elmenhorst J, Hulpke-Wette M, Barta C, et al. (2015): Percentiles for central blood pressure and pulse wave velocity in children and adolescents recorded with an oscillometric device. Atherosclerosis 238: 9-16.

27. Tenderenda-Banasiuk E, Wasilewska A, Taranta-Janusz K, Korzeniecka-Kozerska A (2013): Asymmetric and symmetric dimethylarginine in adolescents with hyperuricemia. Dis Markers 35: 407-412.

28. Takaya J, Tanabe Y, Kuroyanagi Y, Kaneko K (2015): Asymmetric dimethylarginine is negatively correlated with hyperglycemia in children. Endocr J 62: 551-556.

29. Zharikov S, Krotova K, Hu H, et al. (2008): Uric acid decreases NO production and increases arginase activity in cultured pulmonary artery endothelial cells. Am J Physiol Cell Physiol 295: 1183-1190.

30. Zoccali C, Maio R, Mallamaci F, et al. (2006): Uric acid and endothelial dysfunction in essential hypertension. J Am Soc Nephrol 17: 1466-1471.

31. Yilmaz MI, Sonmez A, Saglam M, et al. (2008): ADMA levels correlate with proteinuria, secondary amyloidosis, and endothelial dysfunction. J Am Soc Nephrol 19: 388-395.

32. Hörster I, Weigt-Usinger K, Carmann C, et al. (2015): The $\mathrm{L}$-arginine/NO pathway and homoarginine are altered in Duchenne muscular dystrophy and improved by glucocorticoids. Amino Acids 47: 1853-1863.

33. Uchida HA, Nakamura Y, Kaihara M, et al. (2006): Steroid pulse therapy impaired endothelial function while increasing plasma high molecule adiponectin concentration in patients with IgA nephropathy. Nephrol Dial Transplant 21: 34753480.

34. Hafezi-Moghadam A, Simoncini T, Yang Z, et al. (2002): Acute cardiovascular protective effects of corticosteroids are mediated by non-transcriptional activation of endothelial nitric oxide synthase. Nat Med 2002; 8: 473-479.

35. Fujii H, Kono K, Nakai K, et al. (2014): Renin-angiotensin system inhibitors reduce serum asymmetric dimethylarginine levels and oxidative stress in normotensive patients with chronic kidney disease. Nephron Extra 4: 18-25. 
36. Perticone F, Sciacqua A, Maio R, et al. (2005): Asymmetric dimethylarginine, L-arginine, and endothelial dysfunction in essential hypertension. J Am Coll Cardiol 46: 518-523.

37. Gkaliagkousi E, Gavriilaki E, Triantafyllou A (2018): Asymmetric dimethylarginine levels are associated with augmentation index across nadve untreated patients with different hypertension phenotypes. J Clin Hypertens (Greenwich) 20: 680-685.

38. Protopsaltis I, Foussas S, Angelidi A, et al. (2012): Impact of ADMA, endothelial progenitor cells and traditional cardiovascular risk factors on pulse wave velocity among prediabetic individuals. Cardiovasc Diabetol 2012: 11: 141.

39. Ayer JG, Harmer JA, Nakhla S, et al. (2009): HDL-cholesterol, blood pressure, and asymmetric dimethylarginine are significantly associated with arterial wall thickness in children. Arterioscler Thromb Vasc Biol 29: 943-949.

40. Achan V, Broadhead M, Malaki M, et al. (2003): Asymmetric dimethylarginine causes hypertension and cardiac dysfunction in humans and is actively metabolized by dimethylarginine dimethylaminohydrolase. Arterioscler Thromb Vasc Biol 23: 1455-1459.

41. Päivä H, Kähönen M, Lehtimäki T, et al. (2008): Asymmetric dimethylarginine (ADMA) has a role in regulating systemic vascular tone in healthy subjects: the cardiovascular risk in young Finns study. Am J Hypertens 21: 873-878.

42. Huemer M, Simma B, Mayr D, et al. (2011): Low levels of asymmetric dimethylarginine in children with diabetes mellitus type I compared with healthy children. J Pediatr 158: 602-606. 